\title{
Experimental and Numerical Investigation of Dispersed and Continuous Liquid Film under Boiling conditions
}

\author{
C. Habchi' ${ }^{1,2}$, N. Lamarque ${ }^{3}$, J. Helie ${ }^{3}$ and S. Jay ${ }^{1,2}$ \\ ${ }^{1}$ IFP Energies nouvelles, 1 et 4 avenue de Bois-Préau, 92852 Rueil-Malmaison, France \\ ${ }^{2}$ Institut Carnot IFPEN Transports Energie \\ ${ }^{3}$ Continental Automotive SAS, 1 avenue Paul Ourliac BP 1149, 31036 Toulouse, France \\ ${ }^{*}$ Corresponding author: $\underline{\text { Chaouki.Habchi@ifpen.fr }}$
}

\begin{abstract}
In this work, both experimental and numerical investigations have been carried out in order to improve the modelling of the vaporization of wall liquid-deposits in internal combustion engines. A comprehensive model is suggested for the vaporization of liquid films in the different boiling regimes, including nucleate boiling regime, the Leidenfrost boiling regime, as well as the transition boiling regime occurring between the two latter. This work extends the validity of the Liquid Film Boiling model (Habchi, Oil \& Gas Science and Technology - Rev. IFP, Vol. 65 , No. 2, 2010) for dispersed liquid films that may be formed when a dilute spray impinges a wall. A sub-grid liquid film is indeed considered when the wetted-area is smaller than the wall cell-face area. A sessile droplet model is used to estimate the wall area wetted by the liquid film and whether it is resolved by the grid or located in the sub-grid scale (SGS). In addition, a novel Leidenfrost vaporization model is proposed for spray droplets located near a hot wall. The above vaporisation/boiling models has been implemented in the Large-Eddy simulation (LES) AVBP code. The validation has been carried out using two different experiments. First, the experimental lifetime curve of a sessile droplet (Stanglmaier et al., SAE paper 2002-01-0838) has been used for a quantitative validation in the different boiling regimes. Second, the wall impingement of a heptane spray from a typical gasoline injector from Continental Automotive, has been simulated. The numerical results obtained under boiling conditions, are compared to the liquid film footprints and lifetime provided by the Refractive Index Matching (RIM) experiment which is described in this article.
\end{abstract}

\section{Keywords}

Liquid film, Boiling, Leidenfrost, LES, RIM

\section{Introduction}

In Gasoline Direct Injection (GDI) engines, the impingement of the spray on the walls cannot be avoided in all operating conditions. Therefore, a fine tuning of the operating points involving impact of the spray droplets on the inner walls of the engine must be performed. Computational Fluid Dynamics (CFD) codes are often used for this purpose. However, the modelling of all the spray-wall interactions, including evaporation and the different boiling regimes, remains challenging. In this work, both an experimental and a numerical investigation have been carried out in order to improve the understanding and the modelling of the vaporization of liquid deposits in the boiling regimes. The liquid film sub-models previously developed by the authors [1-5] using a RANS approach have been improved and implemented in the Large-Eddy Simulation (LES) code AVBP [6]. In particular, this work extends the validity of the Liquid Film Boiling (LFB) model [5] for dispersed liquid films or tiny deposits that may be formed when a dilute spray impinges a wall. In addition, experimental measurements have been carried out using the Refractive Index Matching (RIM) method described in Section 1. Next, the different physical sub-models and improvements suggested in the present article are detailed in Section 2. Several sessile droplet test cases have been used in Section 3 for the numerical and physical validations of the sub-models proposed for the different liquid film boiling regimes. Then, a typical GDI spray/wall impact configuration has been used for the validation of the liquid film numerical results (footprints, lifetime ...) under boiling regime.

\section{Experimental setup}

The purpose of the Refractive Index Matching (RIM) experimental set-up is to observe the liquid film generated by one plume of a GDI injector on a transparent plate. This methodology has been introduced by Drake et al. [7] and used by Maligne and Bruneaux [8] to study wall film thickness in an optical Direct injection engine. The principles are depicted in Figure 1. The impingement plate is in quartz and has a known average roughness $R_{a}=15 \mu \mathrm{m}$, which order of magnitude is typical of a piston roughness. Its refractive index, with its roughened surface, is around 1.46 [8], which is close to liquid hydrocarbons studied here (1.385 for n-heptane). When liquid, with a refractive index which closely matches the quartz wall index, is on the window, light scattering is modified. The RIM then easily provides the liquid film footprint and a sufficiently accurate total lifetime. Figure 2 gives a picture of the global set-up. Images are captured by a high-speed CMOS video camera. Exposure time is fixed and equal to $100 \mu \mathrm{s}$ and the frame rate is up to $10^{4} \mathrm{fps}$. The objective on the camera has a fixed focal length of $60 \mathrm{~mm}$ and for 
this study; the $\mathrm{f}$-number is $\mathrm{f} / 8$. Resolution is then $83.3 \mu \mathrm{m} /$ pixel. The quartz plate $(50 \mathrm{~mm}$ diameter) is put on an aluminium hollow cylinder (to allow visualization from below, with a flat mirror), which is heated with an electrical collar, driven by a PID controller, that ensures the quartz window has a known and constant temperature. Temperature is measured with a thermocouple located on the quartz plate, as depicted on Figure 2. The quartz window is enlightened with one light guide (diameter: $1 \mathrm{inch}$ ) connected to a $150 \mathrm{~W}$ continuous light illuminator (halogen lamp). Fuel n-heptane is injected with a GDI 3-holes nozzle.

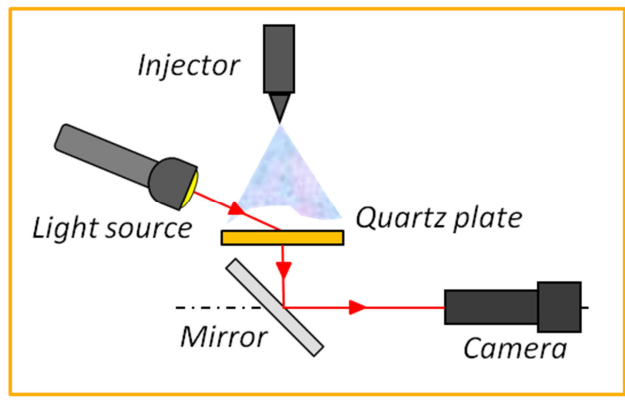

Figure 1. Sketch of the RIM experimental set-up.

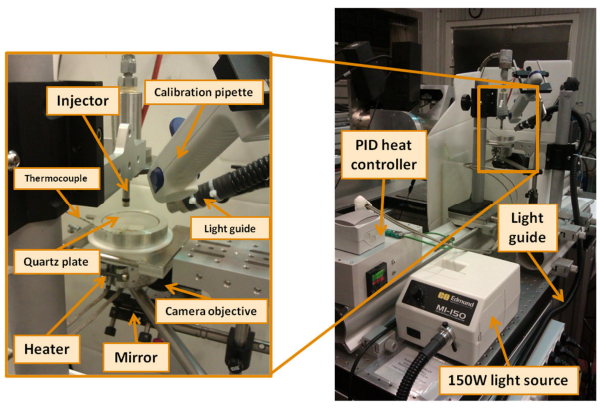

Figure 2. Pictures of the experimental set-up.

As explained in [9], despite the fine calibration, the liquid film thickness is very difficult to be accurately measured. Indeed, it is most often underestimated. Many factors are modifying local and global light scattering, as bubbles (entrapped air) and waves on the film surface, as well as undesired late and slow droplets which may remain several milliseconds levitating above the film. Therefore, only the footprints of the impinging spray and the estimation of the total lifetime of the liquid film are used for the validation in section 3.3. However, many additional useful observations have been made available by our RIM experiments. For instance, it is important to note the existence of a lot of isolated liquid spots all around the continuous liquid film, particularly visible at cold conditions. As they are thin, the associated mass is small in comparison with the central liquid film. Moreover, as their contact line length/surface ratio is high, they then vaporize very fast. The above remark highlights the huge difficulties of the modelling of liquid films (i.e. its formation, evaporation or boiling) at the sub-grid scale (SGS), especially during the impingement of dilute sprays. The main features of the modified SGS LFB model are described in Section 2.2. The validation of such modification is discussed in Section 3. In addition, Figure 3 shows that air can be entrapped during the spray-wall impact in our experiments. Very small bubbles are entrapped in the wall (quartz) troughs as depicted in the zoom of Figure $3(b)$. Those bubbles can survive dozens of milliseconds after end of injection. They can act as first nucleus in boiling conditions.

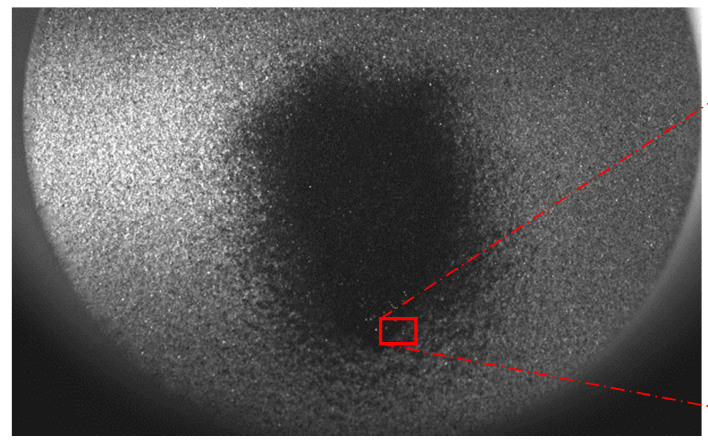

Figure 3(a) Liquid film for : Injection pressure $=100 \mathrm{bar}$ Average roughness $\mathrm{Ra}=15 \mu \mathrm{m}-\mathrm{Ti}=1500 \mu \mathrm{s}-\mathrm{Tw}=30^{\circ} \mathrm{C}$. Image size $(608,376)$ pixel, $83.3 \mu \mathrm{m} /$ pixel.

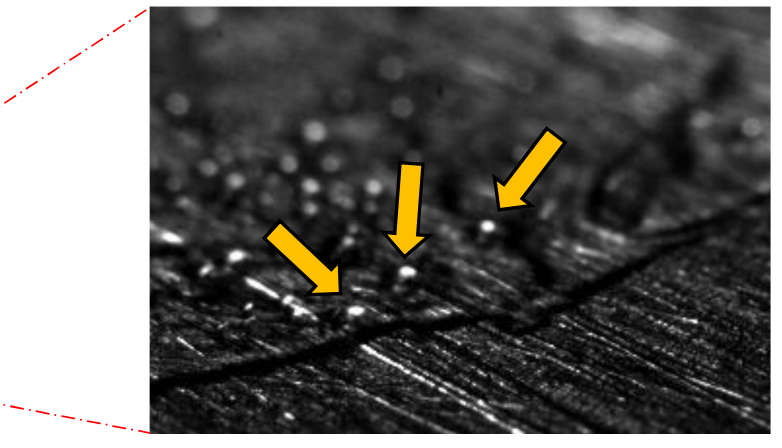

Figure 3(b) Highly zoomed visualisation of a wall film generated by a GDI injector. Image extracted from a highspeed sequence. Some bubbles are shown by orange arrows

\section{Fundamental of boiling of thin films and models description}

For the modelling of a liquid film formed on the surface of a combustion chamber, heat transfer and phase changes are of primary importance. One may distinguish four regimes of vaporization in the literature [10,11] which can be classified according to the superheat degree $\Delta T_{w, s a t}=\left(T_{w}-T_{\text {sat }}\right)$ and uses as limits for these regimes, the saturation temperature $T_{s a t}$, the Nukiyama temperature $T_{N}$ and the Leidenfrost temperature $T_{L}$. $T_{N}$ corresponds to the point of maximum heat flux ( $\mathrm{CHF}$ in boiling curves) or to the minimum point of the lifetime curve (Figure 4); and $T_{L}$ corresponds to local minimum of the curve of the heat flux or to the maximum of the 
lifetime curve separating the Leidenfrost regime IV and boiling regime III, depicted in Figure 4 . These critical temperatures depend on the properties of the wall (roughness, presence of impurity, wettability, thermal conductivity, specific heat, etc.) as well as on gravity, gas pressure, liquid flow rate, etc. (see [5] for a complementary bibliographical review. These critical temperatures may also be determined experimentally from the boiling or lifetime curve of a droplet as depicted in Figure 4 obtained by measuring the total time that it takes to a droplet to completely evaporate after it has been gently deposited on a hot wall [12] :

Regime I, complete wetting regime $\left(T_{w}<T_{\text {sat }}\right)$

The sprays, impinging a wall having a $T_{w}$ smaller than $T_{\text {sat }}$, always form a liquid film which evaporates slowly. In this regime, the evaporation rate strongly depends on the turbulence level in the ambient gas $[3,4,13,14]$.

Regime II, nucleate boiling regime $\left(T_{\text {sat }}<T_{w}<T_{N}\right)$

In this regime, the liquid very close to the wall (e.g. in the thermal boundary layer) is overheated. This leads to the formation of vapour cavities which are born starting from germs (or sites) often hidden in the wall roughness (heterogeneous nucleation) and from the small bubbles are entrapped in the wall troughs as shown in Figure 3(b). While growing, these bubbles may be detached from the wall, especially in case of pool boiling or a raltively thick liquid film. This process of phase change at subcritical conditions prevents the temperature of the liquid $T_{1}$ from exceeding to much its saturation (or spinodal) temperature by consuming most of the heat flux $Q_{w, I}$ transferred to the liquid mainly by the wall. In contrary to pool boiling, the growing vapour cavities cannot be detached from the wall in the case of thin liquid film of interest in this work. Indeed, it has been shown experimentally in [15] that while growing, the vapour cavity diameter increases. After vapour cavities bursting the thin liquid film, tiny droplets are formed as observed in [16] and a dry-spot may appear. In addition, by increasing the superheat degree $\Delta T_{w, s a t}$ in this regime, the number of dry-spots increases. This process leads to a rapid increase of the dry area and the contact line length.

Regime III, transition boiling regime $\left(T_{N}<T_{W}<T_{L}\right)$

This is the transition regime between the nucleate boiling regime and the subsequent Leidenfrost regime. The small vapor cavities and the dry-spots on the wall coalesce progressively and form vapor columns and/or a larger vapor cavity. Indeed, several authors $[11,17,18]$ explained the decrease of the heat flux provided by the wall and the increase of the droplet lifetime, by the coalescence of the vapor cavities formed on the wall. As a consequence, in this transition boiling regime, the heat flux passes more and more through a vapor cushion between the wall and the liquid, and therefore strongly increases the droplet lifetime (Figure 4) with increasing $T_{w}$.

Regime IV, Leidenfrost or "film boiling" regime $\left(T_{w}>T_{L}\right)$

In this regime, there is no liquid film formation on the wall. The impingement always results in the rebound/splashing of the impinging spray droplets. This behavior has been explained by an intense heating of the droplets front, which leads to the formation of a vapor cushion and prevents the contact of the liquid with the wall. In this condition, the wall heats the vapor cushion which in its turn heats the droplets generated by the thermal instabilities during the transition boiling regime. Therefore, the heat flux between the wall and the spray droplets through the vapor cushion must be accounted for in the energy balance in droplets evaporation models. Obviously, this additional heat flux vanishes when the droplet goes far from walls. This additional heat flux will be shown to be of primary importance for the correct prediction of the vaporization lifetime of a sessile droplet in Section 3.1 .

\subsection{Modelling scope}

The modelling scope of this work is the implementation in AVBP [6] of a comprehensive set of models for liquid film boiling. The convective liquid film evaporation, prevailing in regime I, is described by Desoutter et al. [3,4]. The LFB model proposed in [5] is summarized, along with the following improvements:

a) A sub-model for a more accurate sub-grid-scale (SGS) modelling of liquid film boiling.

b) A modified spray evaporation model for droplets near hot walls, particularly for those under the Leidenfrost regime vaporization.

In addition, the wall film droplets formed near the end of the boiling transition regime are released and become free/spray droplets as soon as $T_{L}$ becomes smaller than $T_{w}$ (see the schematics illustrating the boiling states near the limits of the boiling regimes in Figure 4 and 5). It is also worth noting that the spray/wall interaction models such as the rebound or splashing model will not be discussed in this article since they are available elsewhere [19]. In addition, the reader may refer for instance to [20] for the estimation of $T_{N}$ and $T_{L}$.

\subsection{Thin liquid film boiling models}

Il well known that evaporation takes place mostly at the meniscus of a liquid film. According to the LFB model [5], the boiling phenomenon may therefore be modelled using the following two main parameters:

- $\quad$ The dry fraction of liquid film area due to boiling (i.e. vapour cavities and dry-spots), denoted $\boldsymbol{a}_{\boldsymbol{d r y}}$; 
- The solid-liquid-vapour contact line length density (index clld) of the liquid film, denoted $\boldsymbol{k}_{\text {clld }}$.

According to each boiling regime main characteristics, the behaviour of the above two parameters have been correlated to the wall temperature as depicted in Figure 5. $a_{\text {dry }}$ varies inevitably between 0 and 1 when the wall temperature increases from $T_{\text {sat }}$ to $T_{L}$ (depicted in red color in Figure 5 for the case of iso-octane), respectively. Moreover, the experimental observations mentioned above showed that $a_{d r y}$ increases quickly during the nucleate boiling regime. It reaches a value of about 0.7 to 0.9 when $T_{w} \approx T_{N}$. This behaviour of the function $a_{d r y}\left(T_{w}\right)$ has been expressed by the following correlation:

$$
\boldsymbol{a}_{\text {dry }}=\left[\frac{T_{w}-T_{\text {sat }}}{T_{L}-T_{\text {sat }}}\right]^{\frac{1}{4}}
$$

As for the Solid-liquid-vapour contact lines length density $\boldsymbol{k}_{\text {clld }}\left(T_{w}\right)$ of the liquid film, it is defined as an increasing function from 0 to 1 in the nucleate regime of boiling. Then, it decreases in the transition regime of boiling up to a minimal value, denoted $k_{c l l d}^{\text {min }}$. This behaviour is specified by the following correlations:

$$
\begin{gathered}
k_{\text {clld }}=\frac{\boldsymbol{a}_{\text {dry }}}{\boldsymbol{a}_{\text {dry }}\left[T_{w}=T_{N}\right]} \\
k_{\text {clld }}=\left(1-k_{\text {cld }}^{\text {min }}\right)\left[\frac{T_{L}-T_{w}}{T_{L}-T_{\text {sat }}}\right]^{4}+k_{\text {clld }}^{\text {min }}
\end{gathered}
$$

$$
\begin{aligned}
& \text { when } T_{w} \leq T_{N} \\
& \text { when } T_{N}<T_{w} \leq T_{L}
\end{aligned}
$$

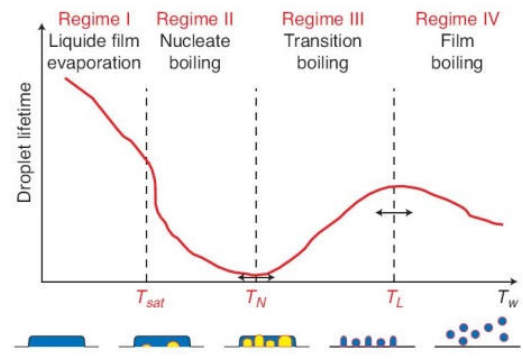

Figure 4. Droplet lifetime curve and schematics illustrating the boiling states near the limits of the boiling regimes (reproduced from [5])

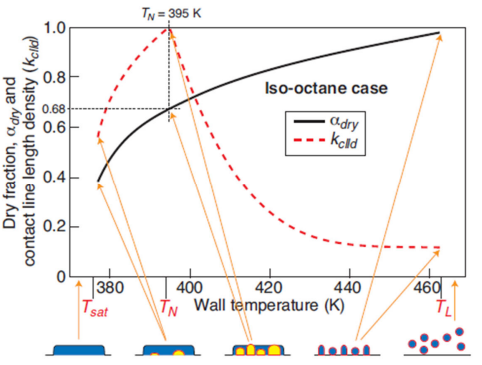

Figure 5. Dry fraction $a_{d r y}$ function and Contact Line Length Density $k_{\text {clld }}$ function drawn for the iso-octane case at atmospheric pressure. The schematics illustrate the boiling states near the limits of the boiling regimes (reproduced from [5]).

For the boiling phenomenon, instantaneous thermodynamic equilibrium is assumed (i.e. no metastable state is permitted). This assumption makes sense for liquid film already including bubbles even in cold conditions, as depicted in Figure 6 . Therefore, the vaporized mass $\dot{M}_{v}$ may be calculated by the following energy balance:

$$
\dot{M}_{v}=\frac{\left[\beta_{1}\left(1-\boldsymbol{a}_{\boldsymbol{d r y}}\right) Q_{w, l}+\beta_{2} \boldsymbol{a}_{\boldsymbol{d r y}} Q_{w, v, l}\right] S_{f i l m}}{L_{\text {sat }}}
$$

where $L_{s a t}$ is the liquid latent heat of vaporization at $T_{s a t .} Q_{w, l}$ is the heat flux per unit area between the wall and the liquid in direct contact. It is calculated by Equation (4) where $\lambda_{l, s a t}$ is the thermal conductivity of the liquid at $T_{s a t}$ and $\delta_{t h}$ has been estimated in [5] of the order of few microns. $Q_{w, v, l}$ is the heat flux per unit area between the wall and the liquid through the vapour cushion. It is the driving heat flux compared to the former in transition boiling regime III, particularly when $T_{w}$ tends towards $T_{L}$. The wall heats the vapour cushion which in its turn heats the liquid. It is calculated by Equation (5) where $\lambda_{v, s a t}$ is the thermal conductivity of the vapour at $T_{\text {sat. }}$ The area $S_{\text {film }}$ is obviously equal to the wall cell-face for a resolved liquid film. But, it is unknown for the tiny liquid film, such as the one depicted around the main footprint of the continuous liquid film in Figure 6(a). In this case, the model of Nagaoka [21] is used for the estimation of the SGS liquid film area, $S_{f i l m}$. In addition, this model has proved sufficiently accurate for the estimation of the average film thickness, $h_{\text {film }}$ for the SGS modelling of non-resolved liquid film transport and vaporisation. Equation (3) also includes two additional coefficients: $\beta_{1}$ represents the fraction of the wetted area $\left(1-\boldsymbol{a}_{d r y}\right)$ where most liquid vaporization is produced (i.e. area of the meniscus); whereas $\beta_{2}$ represents the fraction of $\boldsymbol{a}_{\boldsymbol{d r y}}$ where the liquid film is separated from the wall by a vapour cushion. In our previous work [5], the thickness of the vapour cushion has been correlated to the ambient pressure in accordance with the experimental observations of Temple-Pediani [22]. 


$$
\begin{gathered}
Q_{w, l}=\lambda_{l, s a t} \frac{\left(T_{w}-T_{s a t}\right)}{\delta_{t h}} \\
Q_{w, v, l}=\lambda_{v, s a t} \frac{\left(T_{w}-T_{s a t}\right)}{\delta_{v}} \quad \text { with } \quad \delta_{v}=10^{-4}\left(\frac{P}{P_{0}}\right)^{2}
\end{gathered}
$$

\subsection{Droplets vaporisation near hot walls}

In the AVBP code [6], a typical spray evaporation model, named SIGLE is used. It is a modified Abramzon and Sirignano model [23]. In this work, another modification of this model is carried out in order to account for an additional heat flux coming from hot walls. Indeed, this complementary heat flux is necessary when the spray is close to hot walls, especially in the Leidenfrost regime. Such heat flux is suggested here under the following form:

$$
Q_{w}=\lambda_{g} \frac{\left(T_{w}-T_{d}\right)}{\delta_{g}} S_{d}
$$

Where $T_{d}$ is the droplet temperature and $\delta_{g}$ (respectively $\lambda_{g}$ ) is the thickness (respectively thermal conductivity) of the gas cushion between the wall and the droplet. $\delta_{g}$ is computed as the normal distance to the wall of the droplet centre, minus its radius. Note that this distance cannot be smaller than $\delta_{v}$ given by Equation (5). $\lambda_{g}$ is estimated at a temperature equal to $T_{d}+\left(T_{g}-T_{d}\right) / 3$. Finally, since spray droplets in internal combustion engine are small, the area $S_{d}$ is computed simply as the half of the droplet surface facing the wall. The flux given by Eq. (6) is added to the heat flux coming from the gas phase in the SIGLE droplets evaporation model.

\section{Models validation}

The different models suggested in this paper have been implemented in the LES code AVBP [6]. The validation is carried out using two different experiments. First, the experimental lifetime curve of a sessile droplet from [12] is used for a quantitative validation in the different boiling regimes at resolved and SGS numerical conditions. Second, the wall impingement of a heptane spray from a prototype GDI injector from Continental Automotive, has been simulated. The numerical results obtained under boiling conditions, are compared to the liquid film footprints and lifetime provided by the RIM experiments described in section 1.

\subsection{Sessile drop test cases : Numerical validations}

In the experiments of Stanglmaier et al. [12], a rather big droplet $\left(V_{0}=5 \mu l\right)$ is deposited on an aluminium heated plate. Once deposited gently on the wall, this droplet spreads and forms a liquid film. In this work, we assumed the droplet after impact takes the form of a cylinder with radius $r_{f}$ and height $h_{f}$. In order to compute $r_{f}$ and $h_{f}$, Nagaoka et al. [21] model is used as described in [5]. A sub-grid liquid film is indeed considered when the obtained area $S_{\text {film }}=\pi r_{f}^{2}$ is smaller than the area $S_{w}$ of the wall-cell face where the liquid film is located. In this case, the SGS liquid film height is also taken equal to $h_{f}$ as this value is more accurate than the one usually computed in most CFD codes as $h_{f}=V_{0} / S_{w}$, which value is often underestimated. The initial conditions given by the Nagaoka et al. [21] model and the physical properties are summarized in Table 1 and Table 2, respectively.

Figure 6 shows the initial liquid film deposited on the bottom wall for the SGS case (a) and resolved case (b). The grid used for case (b) is refined five time more than in case (a). The grid size in case (b) is specified in such a way that the wall-area covered by the liquid film, $S_{f i l m}$ is equal to the sum of the 25 central-cell areas. In addition, 25 droplets have been distributed at the centre of each of these 25 wall-cell faces (Figure 6(b)). The initial droplets size is specified in such a way that the same initial volume is used for both cases (a) and (b). For these two cases, two wall temperatures $T_{\text {wall }}$ equal to $388 \mathrm{~K}$ and $423 \mathrm{~K}$ have been simulated. The first wall temperature is chosen in the middle of the nucleate boiling regime and the second one in the transition boiling regime (see $T_{N}$ and $T_{L}$ in Table 2). The numerical results are depicted in Figure 7. First, the resolved and SGS cases are compared in Figure 7(a) in terms of liquid film volume. The liquid film temporal evolutions are somewhat different. But, their total lifetimes are similar, around $0.6 \mathrm{~s}$ and $4.5 \mathrm{~s}$ for $T_{\text {wall }}$ equal to $388 \mathrm{~K}$ and $423 \mathrm{~K}$, respectively. Second, Figure 7(b) shows that even if the liquid film is initially resolved by the grid in case (b), vaporization/boiling reduces the wetted area relatively quickly and makes its calculation required in the SGS.

Table 1 : Initial conditions for sessile drop test cases using iso-octane

\begin{tabular}{|c|c|c|c|c|c|c|c|}
\hline$V_{0}(\mu)$ & $r_{0}(\mathrm{~mm})$ & $r_{f} / r_{0}$ & $h_{f}(\mu \mathrm{m})$ & $S_{\text {film }}\left(\mathrm{mm}^{2}\right)$ & $P_{\text {amb }}(\mathrm{Pa})$ & $T_{a m b}(\mathrm{~K})$ & $T_{d}$ \\
\hline 5 & 1.061 & 4.32 & 86.9 & 57.6 & $1.01 \times 10^{5}$ & $T_{\text {wall }}$ & $T_{\text {sat }}$ \\
\hline
\end{tabular}




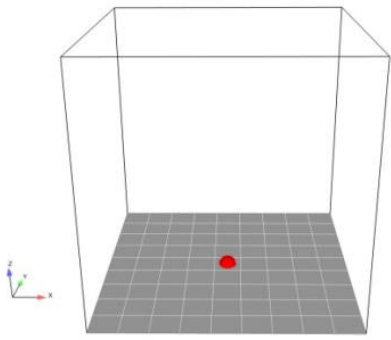

Case (a): Un-resolved liquid film SGS case defined using a single droplet with $r_{0}=1.061 \mathrm{~mm}$, on one wall-cell face.

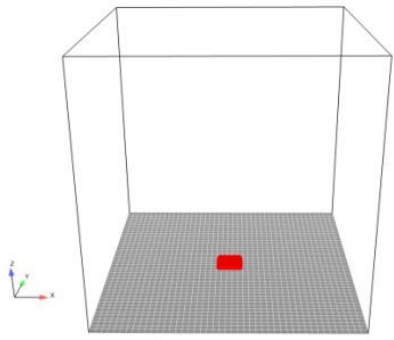

Case (b): Resolved liquid film case defined using 25 droplets with $r_{o}=0.3628 \mathrm{~mm}$, on 25 wall-cell faces.

Figure 6. Initial conditions for SGS and resolved liquid film. The same initial volume is used for both cases (a) and (b).

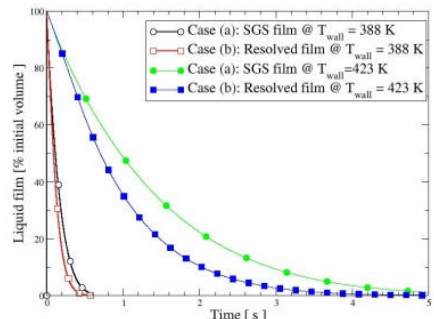

(a) Liquid film volume temporal evolutions.

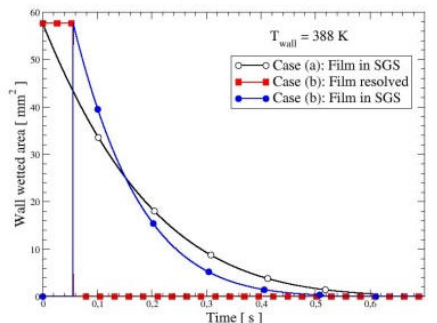

(b) Liquid film areas. SGS vs. resolved liquid film.

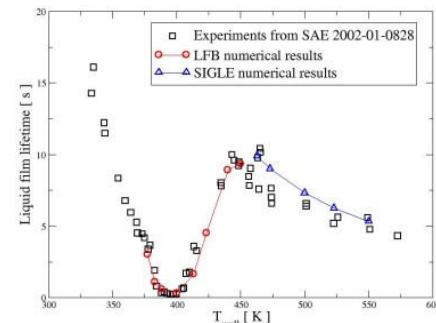

Figure 8. Iso-octane liquid film total lifetime. LFB model is used in nucleate and transition boiling regimes. SIGLE is the droplets vaporisation model used in the Leidenfrost regime.

\subsection{Sessile drop test cases : Experimental validations}

In this subsection, the suggested models in the different boiling regimes are evaluated. Several simulations similar to the above case (a) have been carried out. For the simulations under the Leidenfrost regime, the gas temperature is assumed initially equal to the wall temperature. In this case, the sessile droplet is deposited at the wall, at $h_{d, i n i t}=1 \mathrm{~mm}$ with a zero initial velocity. In addition, the sessile droplet initial temperature is assumed equal to $T_{\text {sat }}$. This is true at least on the sessile droplet surface faced to the wall. Note that the gravity acceleration acting towards the wall is switched on. In these conditions, the rebound regime is invoked each time the droplet hits the wall. The numerical results in terms of liquid volume temporal evolutions are depicted in Figure 9. One may see in this Figure the lifetime decrease (increase) in the nucleate and Leidenfrost regimes (in the transition boiling regime) as wall temperature is increasing (decreasing), respectively. A good agreement of these numerical results with the experiments of Stanglmaier et al. [12] in terms of total lifetimes, is shown in Figure 8. Each symbol ( $\circ$ or $\mathbf{\Delta}$ ) in this Figure is the result of a single AVBP simulation. Although the LFB model (see Section 2.2) is obviously very different from the SIGLE vaporization model (see Section 2.3) proposed in this work, the numerical results show a smooth transition between them.

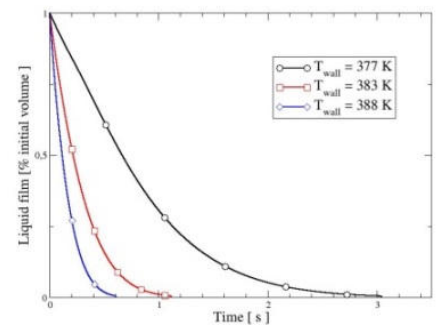

(a) Nucleate boiling regime

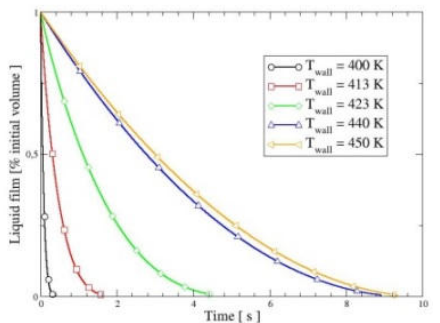

(b) Transition boiling regime

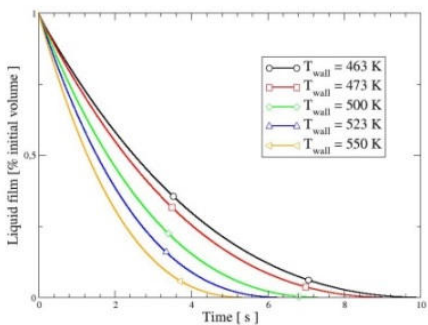

(c) Leidenfrost regime

Figure 9. Liquid volume temporal evolutions from sessile droplets simulations in SGS for 13 different wall temperatures.

\subsection{The GDI spray/wall interaction configuration}

In this section we evaluate the different models suggested in this work for a typical GDI spray impinging a wall. The RIM experimental liquid film results generated by one plume of a GDI injector on a transparent plate are used for comparisons (see section 1). The impingement plate is in quartz and has a known average roughness 
$R_{a}=15 \mu \mathrm{m}$. It is located $40 \mathrm{~mm}$ from the nozzle. The computational domain hexahedral grid is shown in Figure 10(a). It consists of six million nodes. A refined zone has been provided to suitably describe the coupling of the gas with the spray and the liquid film. The cell size in the refined region is $25 \mu \mathrm{m}$. Outlet boundary conditions are specified for the four lateral faces of the computational domain. The bottom face is the heated wall at $T_{\text {wall }}=423 \mathrm{~K}$ for which an isothermal wall law is specified. However, the top wall is assumed at ambient temperature, $T_{a m b}=323 \mathrm{~K}$ for which a free slip isothermal wall is specified. In addition, Figure $10(\mathrm{~b}-\mathrm{c})$ shows the impinging $\mathrm{n}$ heptane spray configuration. Relatively to the vertical direction, the spray axis have an angle of $31^{\circ}$ in the $(x, z)$ plane. The rest of the parameters is given in Table 3. Of course, the spray injection model and the spray-wall interaction and the liquid film formation models including the rebound/splashing phenomena are very important for this configuration. Here, the DVI injection model [24] with an intact liquid core length equal to $2 \mathrm{~mm}$ is used for the numerical simulation of the injection process. The liquid is injected using a given trapezoidal injection rate assuming opening and closing periods equal to $0.3 \mathrm{~ms}$. A velocity coefficient equal to 0.7 is also used in order to take into account the momentum lost inside the nozzle. Moreover, one hundred-thousand parcels have been used for the discretized injected mass $\left(m_{i n j}=3.25 \mathrm{mg}\right)$. With the DVI model, a Rosin-Rammler distribution is specified with a Sauter Mean Diameter, $S M D=12 \mu m$ and a parameter, $q=2.2$. In addition, the $S A B$ secondary breakup model [25] is used, while assuming no-collision between the droplets in the present LES. Spray droplets evaporation is performed using the modified SIGLE model (see Section 2.3). For the spray-wall interaction and liquid film formation models, in-house IFPEN models [19] have been used. Figure 11 shows a fairly good comparison of the LES footprints results relative to the experimental RIM images. In addition, the evaporation rate in the transition boiling regime of $n$-heptane (since $T_{N}<T_{\text {wall }}<T_{L}$ ) is well predicted as the total lifetime of the liquid film is very close to the experimental value, $70 \mathrm{~ms}$.

Table 3 : Injection and physical parameters

\begin{tabular}{|c|c|c|c|c|c|c|}
\hline$P_{a m b}(\mathrm{~Pa})$ & $T_{a m b}(\mathrm{~K})$ & $\rho_{\text {heptane }}\left(\mathrm{kg} / \mathrm{m}^{3}\right)$ & $T_{\text {wall }}(\mathrm{K})$ & $T_{\text {sat }}(\mathrm{K})$ & $T_{N}(\mathrm{~K})$ & $T_{L}(\mathrm{~K})$ \\
\hline $1.01325 \times 10^{5}$ & 323 & 688 & 423 & 368 & 390 & 456 \\
\hline \hline$P_{\text {inj }}(\mathrm{bar})$ & $m_{\text {inj }}\left(10^{-6} \mathrm{~kg}\right)$ & $t_{\text {inj }}(\mathrm{ms})$ & $d_{\text {nozzle }}(\mu \mathrm{m})$ & $S M D_{\text {inj }}(\mu \mathrm{m})$ & Cone angle $(9)$ & $T_{\text {inj }}(\mathrm{K})$ \\
\hline 200 & 3.25 & 1.36 & 200 & 12 & 12.5 & 300 \\
\hline
\end{tabular}

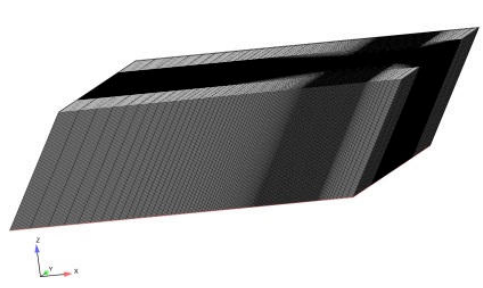

$$
\text { Time }=0.0007 \mathrm{~s}
$$

$$
\text { Time }=0.0014 \mathrm{~s}
$$

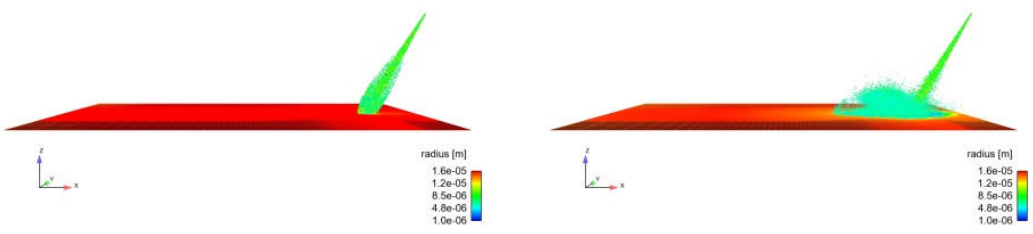

Figure 10. (Left) Mesh (6Mi nodes) and spray orientation (centre) before (right) after impingement.
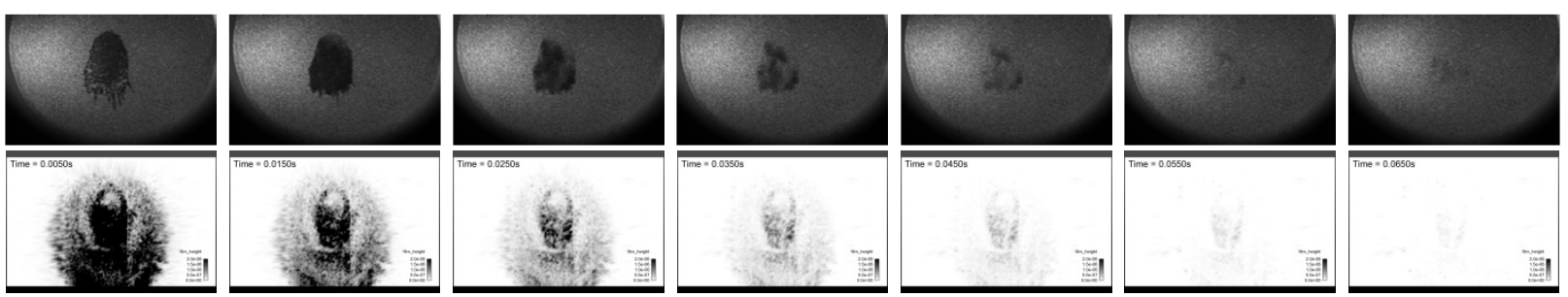

Figure 11. Experimental (top) and LES (bottom) liquid film footprints every $10 \mathrm{~ms}$ starting from $5 \mathrm{~ms}$.

\section{Conclusions}

Two fundamental improvements for the liquid film boiling modelling have been suggested in this article. First, a model allowing the simulation of resolved and sub-grid liquid film boiling is proposed. Second, a modified droplets evaporation model is proposed and applied to the vaporization of droplets located near hot walls, especially for the Leidenfrost boiling regime. These models have been implemented in AVBP and applied to the simulation of the vaporization of an iso-octane sessile droplet in the nucleate boiling regime, the transition boiling regime as well as in the Leidenfrost boiling regime. In addition, RIM experiments have been performed and used for the overall validation the suggested models. The obtained LES results have proved to compare well with our experiments which are of a big interest in several devices like in GDI engines. 


\section{Acknowledgements}

This work has been supported by the French ANR agency in the framework of the project ASTRIDE (reference ANR-12-VPTT-07). It was granted access to the High Performance Computing (HPC) resources of CCRT under the allocations x20162b6139 made by GENCI (Grand Equipement National de Calcul Intensif).

\section{References}

[1] Foucart H., Habchi C., Le Coz J.-F., and Baritaud T., 1998,"Development of a Three Dimensional Model of Wall Fuel Liquid Film for Internal Combustion Engines," SAE paper N`980133.

[2] Habchi C., and Foucart H., 2000,"Multidimensional modelling of gasoline spray impingement and liquid film heat transfer and boiling on heated surfaces," ICLASS Conference Pasadena Proceedings.

[3] Desoutter G., Habchi C., Cuenot B., and Poinsot T., Single component liquid film evaporation model development and validation using direct numerical simulations, 10th ICLASS Conference, Kyoto, Japan, 28/08 - 01/09/2006.

[4] G. Desoutter, C. Habchi, B. Cuenot, and T. Poinsot, 2009,"DNS and modeling of the turbulent boundary layer over an evaporating liquid film," International Journal of Heat and Mass Transfer, 52(25-26), pp. 6028-6041.

[5] Habchi C., 2010,"A Comprehensive Model for Liquid Film Boiling in Internal Combustion Engines," Oil \& Gas Science and Technology - Rev. IFP Energies nouvelles, 65(2), pp. 331-343.

[6] IFPEN-CERFACS LES code,"The AVBP HandBook: http://www.cerfacs.fr/avbp7x/handbook.php (2017),"

[7] Drake M. C., Fansler T. D., Solomon A. S., and Szekely G. A., 2003,"Piston Fuel Films as a Source of Smoke and Hydrocarbon Emissions from a Wall-Controlled Spark-Ignited Direct-Injection Engine," SAE Technical Paper 2003-01-0547.

[8] Maligne D., and Bruneaux G., 2011,"Time-Resolved Fuel Film Thickness Measurement for Direct Injection SI Engines Using Refractive Index Matching," SAE Technical Paper 2011-01-1215.

[9] N. Lamarque, C. Schmit, and J. Hélie, Eds., 2016. Refractive Index Matching Method to Study Gasoline Spray-Wall Impingement:: 9th ICMF, Florence, Italy, 22 - 27 May 2016, Florence, Italy.

[10] Rein M., Ed., 2002. Drop-surface interactions, Springer, Wien, New York.

[11] Dhir V. K., 1998,"Boiling Heat Transfer," Annu. Rev. Fluid Mech., 30(1), pp. 365-401.

[12] Stanglmaier R. H., Roberts C. E., and Moses C. A., 2002,"Vaporization of Individual Fuel Drops on a Heated Surface: A Study of Fuel-Wall Interactions Within Direct-Injected Gasoline (DIG) Engines," SAE Technical Paper 2002-01-0838.

[13] O'Rourke P. J., and Amsden A. A., 2000,"A Spray / Wall Interaction Submodel for the KIVA-3 Wall Film Model," SAE Technical Paper 2000-01-0271.

[14] G. Desoutter, B. Cuenot, C. Habchi, and T. Poinsot, 2005,"Interaction of a premixed flame with a liquid fuel film on a wall," Proc. of the Combust. Inst., 30(1), pp. 259-266.

[15] Gong S., Ma W., and Gu H., 2014,"An experimental investigation on bubble dynamics and boiling crisis in liquid films," International Journal of Heat and Mass Transfer, 79, pp. 694-703.

[16] Bertola V., and Sefiane K., 2005,"Controlling secondary atomization during drop impact on hot surfaces by polymer additives," Phys. Fluids, 17(10), p. 108104.

[17] NISHIO S., and TANAKA H., 2002,"Advanced Fluid Information. Simplified Model Predicting Contact-LineLength Density at Critical Heat Flux Based on Direct Observation of Boiling Structure," JSME Int. J., Ser. B, 45(1), pp. 72-78.

[18] Nigmatulin B. I., Vasiliev N. I., and Guguchkin V. V., 1993,"Interaction between liquid droplets and heated surface," Wärme- und Stoffübertragung, 28(6), pp. 313-319.

[19] C. Habchi, 2005,"Modélisation de l'interaction spray-film liquide par une approche lagrangienne incluant splashing et évaporation," report №58472, IFPEN.

[20] C. Habchi, New Correlations for Leidenfrost and Nukiyama Temperatures with Gas Pressure Application to Liquid Film Boiling Simulation: 23rd ILASS - Europe, Brno, Czech Rep, 6-9 Sept 2010.

[21] Nagaoka M., Kawazoe H., and Nomura N., Eds., 1994. Modeling Fuel Spray Impingement on a Hot Wall for Gasoline Engines: SAE Technical paper 940525

[22] R. W. Temple-Pediani, 1969, "Fuel Drop Vaporization under Pressure on a Hot Surface," Proceedings of the Institution of Mechanical Engineers, 184(1), pp. 677-696.

[23] B. Abramzon, and W.A. Sirignano, 1989,"Droplet vaporization model for spray combustion calculations," International Journal of Heat and Mass Transfer, 32(9), pp. 1605-1618.

[24] C. Habchi, and G. Bruneaux, Eds., 2012. LES and Experimental investigation of Diesel sprays: ICLASS, Heidelberg, Germany, 2-6 sept, 2012.

[25] Habchi C., 2012,"The energy Spectrum Analogy Breakup (SAB ) model for the numerical simulation of sprays," ATOMIZATION AND SPRAYS, 21(12), pp. 1033-1057. 